\title{
Hepatoma-derived growth factor-2 is highly expressed during development and in spinal cord injury
}

\author{
ZERUI ZHUANG ${ }^{1 *}$, GUOLONG MEI $^{2 *}$, WEIDONG LIU $^{3}$, YUCHUN CHEN $^{2}$, \\ JICAN ZENG $^{2}$, WEI ZHANG ${ }^{2}$, GUANFENG YAO ${ }^{2}$ and XINJIA WANG ${ }^{2}$ \\ Departments of ${ }^{1}$ Neurosurgery, ${ }^{2}$ Spinal Surgery and ${ }^{3}$ Infectious Diseases, The Second Affiliated Hospital, \\ Shantou University Medical College, Shantou, Guangdong 515041, P.R. China
}

Received October 12, 2014; Accepted June 18, 2015

DOI: $10.3892 / \mathrm{mmr} .2015 .4195$

\begin{abstract}
Hepatoma-derived growth factor-2 (HDGF-2) is expressed in neurons, astrocytes and oligodendrocytes of the adult mouse brain. However, it has remained elusive whether HDGF-2 is expressed in the spinal cord and is involved in the its development and repair. In the present study, the expression of HDGF-2 was investigated in rat spinal cords at different developmental stages and following spinal cord injury (SCI). Protein levels of HDGF-2 were examined using western blot analysis, while the distribution pattern and cell populations of HDGF-2 protein expression were characterized using immunohistochemistry. Western blot analysis demonstrated that the levels of HDGF-2 protein expression were the greatest in the spinal cord on embryonic day 19, and were also highly expressed in rat spinal cords on post-natal day 7 (P7); however, they were low at P14 and not detectable at two months. HDGF-2 expression was significantly upregulated in the embryonic spinal cord and injured spinal cord. By contrast, the expression of HDGF-2 was low in uninjured adult spinal cords. HDGF-2 expression in the fetal rat spinal cord and injured spinal cord was significantly higher than that in uninjured adult spinal cord tissues $(\mathrm{P}<0.05)$. The number of cells positive for HDGF-2 was $141 \pm 62,107 \pm 33$ and $92 \pm 18$ at days 1, 21 and 45 following SCI, respectively, as opposed to $50 \pm 9$ in uninjured rats, and a significant difference was identified between the different time-points following SCI $(\mathrm{P}<0.01)$. In conclusion, the overexpression of HDGF-2 in the embryonic
\end{abstract}

Correspondence to: Professor Xinjia Wang, Department of Spinal Surgery, The Second Affiliated Hospital, Shantou University Medical College, 69 Dongxiabei Road, Shantou, Guangdong 515041, P.R. China

E-mail: xj.wang2000@163.com

*Contributed equally

Key words: hepatoma-derived growth factor-2, spinal cord injury, embryonic and adult spinal cord, immunohistochemistry, western blot spinal cord and injured spinal cord may be involved in fetal spinal cord development and repair of SCI, respectively.

\section{Introduction}

Hepatoma-derived growth factors (HDGFs) are novel multifunctional growth factors that were first purified from the HuH-7 hepatoma cell line (1) and rat metanephrotic mesenchymal cells (2). Subsequently, five HDGF-associated proteins (HRP1-4) and lens epithelium-derived growth factor (LEDGF) were identified. HRP1-4 and LEDGF constitute the HDGF family. Members of the HDGF family are expressed in a broad range of organs, including the brain, testes, lungs, skeletal muscles and the spleen (3-6).

HDGF has mitogenic activity in numerous cell types, including hepatocellular carcinoma (HCC) cells, fibroblasts, endothelial cells, vascular smooth muscle cells, neuronal cells and fetal hepatocytes (1,2,7-9). HDGF is also involved in the development of the kidney, liver and lungs, in addition to cardiovascular differentiation (2,4,5,10-12). HDGF has been suggested to be associated with tumorigenesis, due to the fact that upregulation of HDGF expression has been observed in human gastric cancer (13), non-small-cell lung cancer (14,15), HCC (16), esophageal carcinoma (17) and pancreatic cancer (18) cells. HDGF has additionally been reported to stimulate DNA synthesis and cell proliferation upon entering the nuclei of tumor cells and serve important roles in angiogenesis, tumor relapse, distant metastasis and malignancy (19).

HDGF is a novel trophic factor for motor neurons, promoting neurite extension and the survival of spinal motor neurons in primary cultures, to an extent equivalent to the effects of the well-known ciliary neurotrophic factor and brain-derived neurotrophic factor $(20,21)$. HDGF expression was reported to be increased in spinal motor neurons in a mouse model of motor neuron degeneration and in polyglutamine-tract-binding protein-1 transgenic mice prior to the onset of degeneration (22). In addition, an in vivo study in newborn rats demonstrated that HDGF represses cell death of motor neurons following facial nerve sectioning (22). Although the expression of HDGF in numerous organs has been reported previously, the expression of HDGF-2 in the development and injury of the spinal cord has remained to be 
fully elucidated. It was hypothesized in the present study that HDGF-2 is involved in spinal cord development and spinal cord recovery following spinal cord injury (SCI). The purpose of the present study was to investigate alterations in the expression of HDGF-2 in normal fetal and adult rat spinal cords and in injured spinal cords following spinal cord contusion.

\section{Materials and methods}

The current study was approved by the institutional and licensing committee of Shantou University Medical College (STUMC; Shantou, China).

Animals. A total of 120 Adult Sprague-Dawley rats weighing an average of $250 \mathrm{~g}$ (220-270 g; two months old) were used in the current study (provided by the Experimental Animal Center of STUMC). Adult rats were assigned randomly to the SCI groups (including the day 1, day 21 and day 45 subsequent to SCI groups; $n=30$ for each group) and the normal adult rat group $(n=30)$. Rats at embryonic day $19(E 19 ; n=40)$, post-natal day $7(\mathrm{P} 7 ; n=30)$ and P14 $(n=30)$ were used. Rats were housed in cages (7-8 rats per cage for each group) and maintained under a 12 -h light/dark cycle at $21 \pm 1^{\circ} \mathrm{C}$ and $50 \pm 5 \%$ humidity. All animals were acclimated to their environment and had ad libitum access to tap water and a standard rodent diet. All experimental procedures were performed according to the guidelines of STUMC and were approved by the Medical Animal Care and Welfare committee of STUMC at the Second Affiliated Hospital Shantou University Medical College for the care of animals.

Adult rat model of SCI. Rats were anesthetized by intraperitoneal injection of pentobarbital (40 mg/kg; Beijing Chemical Reagent Company, Beijing, China). Anesthesia was considered complete when animals failed to exhibit hind limb withdrawal in response to a noxious foot pinch. The spinal cords of adult rats were contused extradurally with a 10-g Modified Allen's weight-drop impactor comprising a $10 \mathrm{~g}$ weight dropping $25.0 \mathrm{~mm}$ onto the T9-T10 region of the spinal cord exposed by laminectomy as described previously (23). All animal care and surgical procedures were approved by the institutional animal care and use committee of Shantou University.

Western blot analysis. The spinal cords from the T9-T10 region were dissected from the E19, P7 and adult rats. Cord tissues were suspended in lysis buffer (Cell Signaling Technology, Danvers, MA, USA) and homogenized in a dounce homogenizer (Ningbo SCIENTZ Biotechnology Co., Ltd., Ningbo, China) on ice. Tissue homogenates were centrifuged at $3,948 \times \mathrm{g}$ for $10 \mathrm{~min}$ at $4^{\circ} \mathrm{C}$ and then stored at $-30^{\circ} \mathrm{C}$. Protein concentrations were determined by a Bio-Rad protein assay kit II (Bio-Rad Laboratories, Inc., Hercules, CA, USA) according to the manufacturer's instructions. For the western blot analysis, $20 \mu \mathrm{l}$ of each sample was separated by $12 \%$ SDS-PAGE and proteins were transferred to polyvinylidene difluoride membranes. Blots were blocked with $5 \%$ non-fat dry milk in Tris-buffered saline for $1 \mathrm{~h}$ at room temperature and then incubated with 1:200 diluted monoclonal rabbit antibodies against HDGF-2 (cat. no. sc-292373; Bioss Biotech Co., Ltd, Beijing, China) overnight at $4^{\circ} \mathrm{C}$.

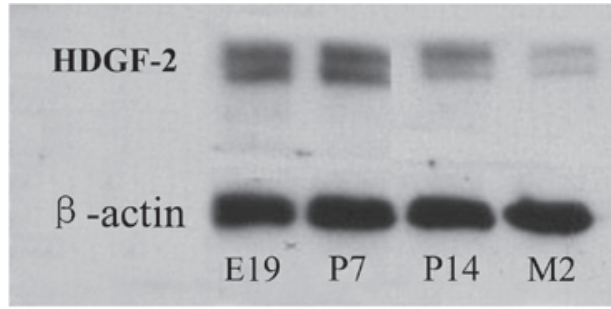

Figure 1. Western blot analysis of hepatoma-derived growth factor-2 expression in the spinal cord tissue of rats at different developmental stages. E19, embryonic day 19; P7, post-natal day 7; P14, post-natal day 14; M2, two-month-old rats.

Membranes were then processed with horseradish peroxidase-conjugated goat anti-rabbit secondary antibody (1:500; Sigma-Aldrich, St. Louis, MO, USA). Immunoreactive bands were quantified using image analysis software.

Immunohistochemistry. Rats were deeply anesthetized and perfused via cardiac puncture with $0.1 \mathrm{M}$ phosphate-buffered saline (PBS; pH 7.4) and subsequently with $4 \%$ paraformaldehyde in 0.1 M PBS.

The spinal cord was carefully dissected from the T9-T10 region (an 8-mm segment containing the injured epicenter was dissected out in the injury group). Isolated spinal cords were post-fixed by immersion in $4 \%$ paraformaldehyde for $3 \mathrm{~h}$, then cryoprotected by immersion in PBS containing $20 \%$ sucrose (Beijing Chemical Reagent Company) overnight. The tissue segments were then embedded in optimal cutting temperature compound for frozen sections and frozen transverse spinal sections $(10 \mu \mathrm{m})$ were cut with a Leica CM1800 cryostat (Leica Microsystems GmbH, Wetzlar, Germany). Immunohistochemical staining was then performed using an avidin-biotin complex method as described previously $(23,24)$. The rabbit anti-HDGF-2 primary antibody and biotinylated anti-rabbit antibody were diluted to 1:200.

Evaluation of staining. Immunohistochemically-stained tissue sections were evaluated for clinical parameters by three pathologists blinded to the treatment groups. HDGF-2 expression in the nuclei was independently evaluated. Five fields of vision were randomly selected at 400x to count positive cells, and the average number of positive cells was calculated. The criteria for positive staining were as follows: Positive staining in vascular endothelial cell nuclei, 1; staining intensity of HDGF-2 in the nuclei was rated as weak (1), mild (2) and strong (3). Positive HDGF-2 expression was additionally evaluated in the cytoplasm, in which the percentage of brown-yellow cells was scored as negative $<5 \%,(-)$; or positive $5-25 \%,(+) ; 25.1-50 \%,(++) ;>50 \%,(+++)$. The same principle was applied in the assessment of spinal cord anterior horn motor neurons.

Statistical analysis. Values are expressed as the mean \pm standard deviation. Statistical significance was assessed by one-way analysis of variance. Significant differences between the two pairs of groups were assessed by Pearson's $\chi^{2}$ test. Statistical analysis was performed using SPSS 17.0 (SPSS, 
Table I. Correlation between time period subsequent to SCI and expression of hepatoma-derived growth factor-2 (staining intensity) ( $\mathrm{n}=30$ per group).

\begin{tabular}{|c|c|c|c|c|}
\hline \multirow[b]{2}{*}{ Parameter } & \multirow[b]{2}{*}{ Normal } & \multicolumn{3}{|c|}{ SCI } \\
\hline & & 1 day & 21 days & 45 days \\
\hline $\begin{array}{l}\text { Positive score } \\
\text { t-value }\end{array}$ & $50.23 \pm 8.76$ & $\begin{array}{c}141 \pm 61.76 \\
57.07\end{array}$ & $\begin{array}{c}107 \pm 33.28 \\
46.29\end{array}$ & $\begin{array}{c}92 \pm 17.92 \\
20.31\end{array}$ \\
\hline
\end{tabular}

$\mathrm{P}<0.01$ vs. normal group. Values are expressed as the mean \pm standard deviation. SCI, spinal cord injury.

Table II. Correlation between group and cytoplasmic expression of hepatoma-derived growth factor-2.

\begin{tabular}{|c|c|c|c|c|c|c|c|}
\hline \multirow[b]{2}{*}{ Group } & \multirow[b]{2}{*}{$\mathrm{n}$} & \multirow{2}{*}{$\frac{\text { Negative }(\mathrm{n})}{-}$} & \multicolumn{3}{|c|}{ Positive (n) } & \multirow[b]{2}{*}{ Positive rate $(\%)$} & \multirow[b]{2}{*}{ P-value } \\
\hline & & & + & ++ & +++ & & \\
\hline Normal & 30 & 25 & 2 & 2 & 1 & 16.67 & \\
\hline Fetal & 40 & 13 & 5 & 10 & 12 & 67.50 & $<0.05$ \\
\hline SCI & 90 & 20 & 8 & 20 & 42 & 77.78 & $<0.05$ \\
\hline
\end{tabular}

SCI, spinal cord injury.
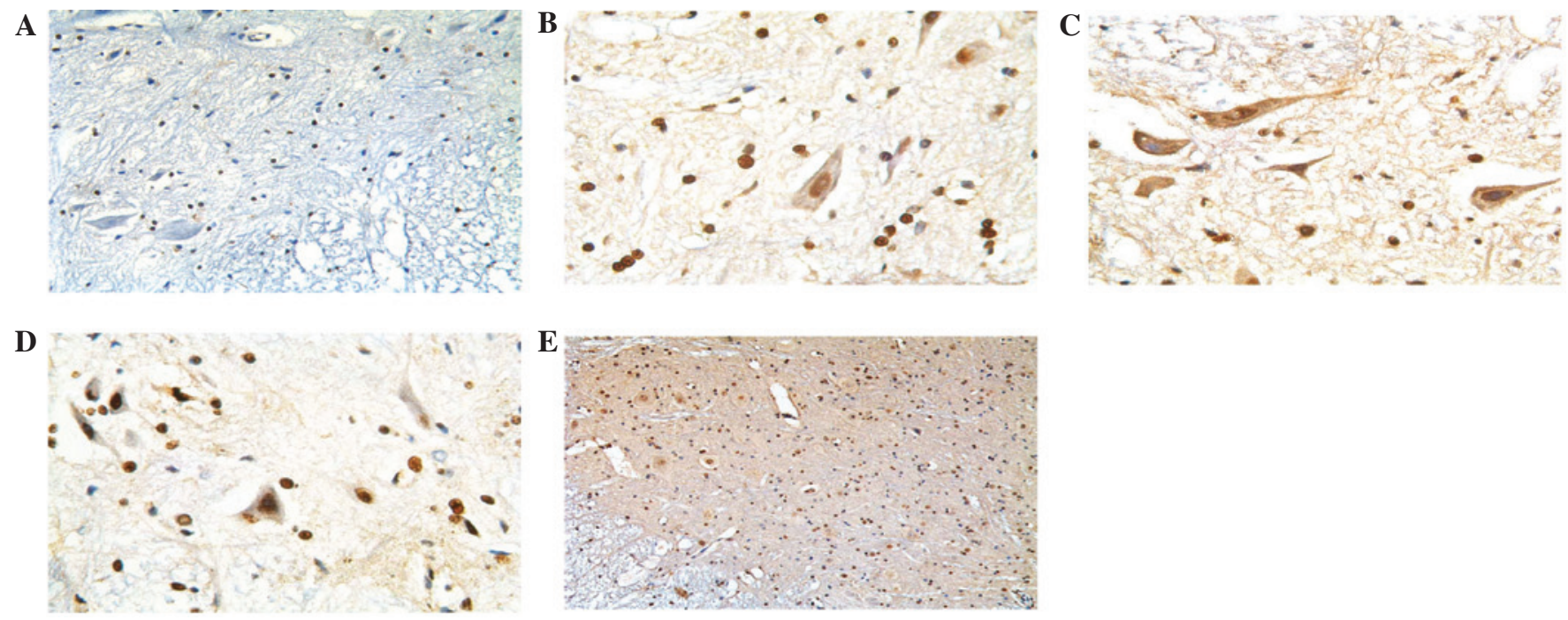

Figure 2. Immunohistochemical avidin-biotin complex staining of the spinal cord tissue in different groups at E19. (A) Normal adult rat spinal cord tissue; (B) Spinal cord tissue at day 1 following SCI; (C) Spinal cord tissue on day 21 subsequent to SCI; (D) Spinal cord tissue at day 45 subsequent to SCI; (E) Spinal cord fetal spinal cord tissue (magnification, $\mathrm{x} 400$ ). SCI, spinal cord injury.

Inc., Chicago, IL, USA). $\mathrm{P}<0.05$ was considered to indicate a statistically significant difference.

\section{Results}

HDGF-2 is highly expressed in spinal cords of neonatal rats. To examine the expression of HDGF-2 during development of the spinal cord, the expression of HDGF-2 was detected in the spinal cord homogenates prepared from rats at different developmental stages using western blot analysis. HDGF-2 protein expression was identified to be highest in the spines of E19 rats and remained high at P7; however, it became weak at P14 and was the lowest at two months (Fig. 1).

In order to identify the distribution pattern of HDGF-2 protein and the cell populations that express HDGF-2, immunohistochemical staining was performed. In the P7 rats, HDGF-2 protein expression was primarily restricted to neurons, astrocytes and oligodendrocytes and it was particularly expressed in motor neurons of the anterior horn. HDGF-2 immunoreactivity was observed at low levels within the white matter. At the sub-cellular level, HDGF-2 was primarily localized within cell nuclei but was additionally detected in the 
cytoplasm (Fig. 2). HDGF-2 was highly expressed in the E19 rat spinal cords, whereas it was weakly expressed in the spinal cords of the two-month-old rats.

In order to deduce the possible role of HDGF-2 in spinal cord development and recovery from SCI, the expression of HDGF-2 was assessed using immunohistochemical s-p staining in embryonic and adult spinal cords and injured spinal cords following SCI. HDGF-2 immunoreactivity was minimally observed in normal adult rat spinal cords (Fig. 2A), however, it was highly expressed in E19 embryonic rat spinal cords (Fig. 2B). As early as one day subsequent to injury, HDGF-2 expression was identified in neurons within the gray matter in the lesion area (Fig. 2C). HDGF-2 was highly expressed until day 21 subsequent to injury (Fig. 2D) and almost declined to basal levels 45 days following injury (Fig. 2E).

HDGF-2 protein was highly expressed in the spinal cords of rat embryos and adult rats at the early stages of SCI. The expression of HDGF-2 at days 1,21 and 45 subsequent to SCI was significantly higher than that in the normal rats $(141 \pm 62$, $107 \pm 32$ and $92 \pm 18$ vs. $50 \pm 9$, respectively). Significant differences between the different time-points following SCI were identified $(\mathrm{P}<0.01)$. Details of the positive HDGF-2 expression observed in the nuclei are presented in Table I and that observed in the cytoplasm are presented in Table II. The percentage of cells stained positive for HDGF-2 was $16.67 \%$ in the spinal cords of normal adult rats, whereas the percentage was significantly increased in fetal rats and SCI rats, with rates of 67.50 and $77.78 \%$, respectively $(\mathrm{P}<0.05)$. The expression of HDGF-2 in the spinal cord following SCI and in fetal rats was significantly higher than that in the normal adult rats $(\mathrm{P}<0.05)$. Pearson's $\chi^{2}$ tests demonstrated that the expression levels of HDGF-2 in the anterior horn motor neurons of SCI rats were different among various post-operative stages $(\mathrm{P}<0.01)$ and the expression levels of HDGF-2 correlated with different time-points following SCI.

\section{Discussion}

HDGF is a novel trophic factor for motor neurons, promoting neurite extension and the survival of spinal motor neurons in primary cultures $(20,21)$. An in vivo study in newborn rats demonstrated that HDGF represses the cell death of motor neurons following facial nerve sectioning (22). Although the expression of HDGF in numerous organs had been previously reported (3-6), the expression of HDGF-2 in the development and injury of the spinal cord has remained elusive. It was hypothesized that HDGF-2 is involved in spinal cord development and spinal cord recovery following SCI. The purpose of the present study was to investigate alterations in the expression levels of HDGF-2 in the spinal cord of fetal and normal adult rats as well as in rats following SCI.

HDGF-2 has previously been demonstrated to have neurotrophic activity $(8,22)$. Abouzied et al $(25)$ reported that the expression levels of HDGF and HDGF-2 are regulated during brain development, with the highest levels around the time-point of birth followed by a decline by post-natal day 9 . In order to gain an improved understanding of the normal organization and development of the human spinal cord, numerous studies have been conducted regarding spinal cord development in lower vertebrates and mammals. For example,
Clowry et al (26) used a restricted set of immunohistochemical markers to follow the development of sensorimotor components of the spinal cord and identified that human fetal spinal cord development during gestational weeks 7.5-17 is similar to the late embryonic/early post-natal period of rodent development (E16.5-P5). During this period, motor neurons segregate into motor columns. Therefore, E19 embryonic rat spinal cords were selected for the present study and it was observed that HDGF-2 was highly expressed in the fetal spinal cord and anterior horn motor neurons in the spinal cord, and that HDGF-2 was predominantly localized in the nuclei. Statistical analysis additionally suggested that the expression of HDGF-2 in the fetal spinal cord was greater than that in the normal spinal cord from protein expression levels, suggesting that HDGF-2 may be involved in the development of the nervous system.

HDGF-2 expression is induced following SCI, particularly during the early phase of recovery from SCI. It has been previously reported that HDGF promotes the proliferation and survival of neurons (27). El-Tahir et al (3) hypothesized that the function of HDGF during neuronal development changes from promoting proliferation to promoting cellular survival. In the present study, it was demonstrated that the HDGF-2 protein expression levels were the highest in the E19 spinal cord and it was also highly expressed in P7 rat spinal cords; however, expression became weak at P14 and was not detectable at two months. HDGF-2 expression in the fetal rat spinal cord and injured spinal cord was higher than that in uninjured adult spinal cord tissues. The expression of HDGF-2 was the highest at the early phases following SCI, suggesting that HDGF-2 may be involved in the early recovery from SCI. Therefore, it was hypothesized that HDGF-2 may be involved in fetal spinal cord development and the repair of damage from SCI.

In conclusion, the present study showed assessed the expression of HDGF-2 in the rat spinal cord at different developmental stages and following SCI. Expression of HDGF-2 following SCI and in fetal rat spinal cord tissues was greater than that in normal adult spinal cord tissues. Expression of HDGF-2 was additionally significantly elevated in the early period following SCI as compared with that in the later stages following recovery from SCI. Thus, the results of the present study suggested that HDGF-2 may be a novel therapeutic substance to aid in the recovery from SCI.

\section{Acknowledgements}

The current study was supported by grants from the Natural Science Foundation of Guangdong Province, China (grant no. S2011010005018) and the Foundation of Guangdong Technology Bureau, China (grant no. 2009B080701040). The authors would like to thank Dr Stanley Lin for his assistance with the revision of the manuscript (Shantou University Medical College, Shantou, China).

\section{References}

1. Nakamura H, Izumoto $\mathrm{Y}$, Kambe H, Kuroda T, Mori T, Kawamura K, Yamamoto H and Kishimoto T: Molecular cloning of complementary DNA for a novel human hepatoma-derived growth factor. Its homology with high mobility group-1 protein. J Biol Chem 269: 25143-25149, 1994. 
2. Oliver JA and Al-Awqati Q: An endothelial growth factor involved in rat renal development. J Clin Invest 102: 1208-1219, 1998.

3. El-Tahir HM, Dietz F, Dringen R, Schwabe K, Strenge K, Kelm S, Abouzied MM, Gieselmann V and Franken S: Expression of hepatoma-derived growth factor family members in the adult central nervous system. BMC Neurosci 7: 6, 2006.

4. Everett AD, Narron JV, Stoops T, Nakamura H and Tucker A: Hepatoma-derived growth factor is a pulmonary endothelial cell-expressed angiogenic factor. Am J Physiol Lung Cell Mol Physiol 286: L1194-L1201, 2004.

5. Mori M, Morishita H, Nakamura H, Matsuoka H, Yoshida K Kishima Y, Zhou Z, Kida H, Funakoshi T, Goya S, et al: Hepatoma-derived growth factor is involved in lung remodeling by stimulating epithelial growth. Am J Respir Cell Mol Biol 30: 459-469, 2004

6. Li SZ, Zhao YB, Cao WD, Qu Y, Luo P, Zhen HN, Chen XY, Yan ZF and Fei Z: The expression of hepatoma-derived growth factor in primary central nervous system lymphoma and its correlation with angiogenesis, proliferation and clinical outcome. Med Oncol 30: 622, 2013

7. Zhou Y, Zhou N, Fang W and Huo J: Overexpressed HDGF as an independent prognostic factor is involved in poor prognosis in Chinese patients with liver cancer. Diagn Pathol 5: 58, 2010.

8. Zhou Z, Yamamoto Y, Sugai F, Yoshida K, Kishima Y, Sumi H, Nakamura $\mathrm{H}$ and Sakoda S: Hepatoma-derived growth factor is a neurotrophic factor harbored in the nucleus. J Biol Chem 279: 27320-27326, 2004

9. Enomoto H, Yoshida K, Kishima Y, Okuda Y and Nakamura H: Participation of hepatoma-derived growth factor in the regulation of fetal hepatocyte proliferation. J Gastroenterol 37 (Suppl 14): 158-161, 2002.

10. Everett AD: Identification, cloning and developmental expression of hepatoma-derived growth factor in the developing rat heart. Dev Dyn 222: 450-458, 2001

11. Enomoto H, Yoshida K, Kishima Y, Kinoshita T, Yamamoto M, Everett AD, Miyajima A and Nakamura H: Hepatoma-derived growth factor is highly expressed in developing liver and promotes fetal hepatocyte proliferation. Hepatology 36: 1519-1527, 2002.

12. Enomoto H, Nakamura H, Liu W, Yoshida K, Okuda Y, Imanishi H, Saito M, Shimomura S, Hada T and Nishiguchi S: Hepatoma-derived growth factor is induced in liver regeneration. Hepatol Res 39: 988-997, 2009.

13. Chang KC, Tai MH, Lin JW, Wang CC, Huang CC, Hung $\mathrm{CH}$, Chen $\mathrm{CH}$, Lu SN, Lee CM, Changchien CS, et al: Hepatoma-derived growth factor is a novel prognostic factor for gastrointestinal stromal tumors. Int J Cancer 121: 1059-1065, 2007.

14. Iwasaki T, Nakagawa K, Nakamura H, Takada Y, Matsui K and Kawahara K: Hepatoma-derived growth factor as a prognostic marker in completely resected non-small-cell lung cancer. Oncol Rep 13: 1075-1080, 2005
15. Ren H, Tang X, Lee JJ, Feng L, Everett AD, Hong WK, Khuri FR and Mao L: Expression of hepatoma-derived growth factor is a strong prognostic predictor for patients with early-stage non-small-cell lung cancer. J Clin Oncol 22: 3230-3237, 2004.

16. Yoshida K, Tomita Y, Okuda Y, Yamamoto S, Enomoto H, Uyama H, Ito H, Hoshida Y, Aozasa K, Nagano H, et al: Hepatoma-derived growth factor is a novel prognostic factor for hepatocellular carcinoma. Ann Surg Oncol 13: 159-167, 2006.

17. Yamamoto S, Tomita Y, Hoshida Y, Morii E, Yasuda T, Doki Y, Aozasa K, Uyama H, Nakamura H and Monden M: Expression level of hepatoma-derived growth factor correlates with tumor recurrence of esophageal carcinoma. Ann Surg Oncol 14: 2141-2149, 2007.

18. Uyama H, Tomita Y,Nakamura H, Nakamori S, Zhang B, Hoshida Y, Enomoto H, Okuda Y, Sakon M, Aozasa K, et al: Hepatoma-derived growth factor is a novel prognostic factor for patients with pancreatic cancer. Clin Cancer Res 12: 6043-6048, 2006.

19. Zhang J, Ren H, Yuan P, Lang W, Zhang L and Mao L: Down-regulation of hepatoma-derived growth factor inhibits anchorage-independent growth and invasion of non-small cell lung cancer cells. Cancer Res 66: 18-23, 2006.

20. Arakawa Y, Sendtner M and Thoenen H: Survival effect of ciliary neurotrophic factor (CNTF) on chick embryonic motoneurons in culture: Comparison with other neurotrophic factors and cytokines. J Neurosci 10: 3507-3515, 1990.

21. Sendtner M, Holtmann B, Kolbeck R, Thoenen H and Barde YA: Brain-derived neurotrophic factor prevents the death of motoneurons in newborn rats after nerve section. Nature 360: 757-759, 1992.

22. Marubuchi S, Okuda T, Tagawa K, Enokido Y, Horiuchi D, Shimokawa R, Tamura T, Qi ML, Eishi Y, Watabe K, et al: Hepatoma-derived growth factor, a new trophic factor for motor neurons, is up-regulated in the spinal cord of PQBP-1 transgenic mice before onset of degeneration. J Neurochem 99: 70-83, 2006.

23. Wang XJ, Kong KM, Qi WL, Ye WL and Song PS: Interleukin-1 beta induction of neuron apoptosis depends on p38 mitogen-activated protein kinase activity after spinal cord injury. Acta Pharmacol Sin 26: 934-942, 2005.

24. Tomlinson A, Appleton I, Moore AR, Gilroy DW, Willis D, Mitchell JA and Willoughby DA: Cyclo-oxygenase and nitric oxide synthase isoforms in rat carrageenin-induced pleurisy. $\mathrm{Br} \mathrm{J}$ Pharmacol 113: 693-698, 1994.

25. Abouzied MM, Baader SL, Dietz F, Kappler J, Gieselmann V and Franken S: Expression patterns and different subcellular localization of the growth factors HDGF (hepatoma-derived growth factor) and HRP-3 (HDGF-related protein-3) suggest functions in addition to their mitogenic activity. Biochem J 378: 169-176, 2004.

26. Clowry GJ, Moss JA and Clough RL: An immunohistochemical study of the development of sensorimotor components of the early fetal human spinal cord. J Anat 207: 313-324, 2005.

27. Bremer S, Klein K, Sedlmaier A, Abouzied M, Gieselmann V and Franken S: Hepatoma-derived growth factor and nucleolin exist in the same ribonucleoprotein complex. BMC Biochem 14: 2, 2013. 